Cahiers

Balkaniques

\section{Cahiers balkaniques}

Hors-série | 2015

Les élites grecques modernes, $\mathrm{XVIII} \mathrm{e}^{\mathrm{X}} \mathrm{XX}^{\mathrm{e}}$ siècles :

identités, modes d'action, représentations

\title{
Kazantzakis, un intellectuel à l'épreuve de la réalité
}

Kazantzakis, an Intellectual confronted to Reality

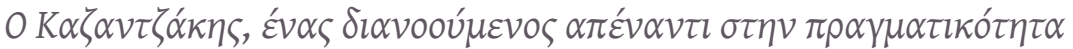

\section{Gunnar De Boel}

\section{OpenEdition}

Journals

Édition électronique

URL : https://journals.openedition.org/ceb/5765

DOI : $10.4000 /$ ceb. 5765

ISSN : 2261-4184

Éditeur

INALCO

Édition imprimée

Date de publication : 1 mars 2015

ISBN : 978-2-85831-224-5

ISSN : 0290-7402

Référence électronique

Gunnar De Boel, « Kazantzakis, un intellectuel à l'épreuve de la réalité », Cahiers balkaniques [En ligne], Hors-série | 2015, mis en ligne le 11 décembre 2015, consulté le 06 juillet 2021. URL : http:// journals.openedition.org/ceb/5765; DOI : https://doi.org/10.4000/ceb.5765

Ce document a été généré automatiquement le 6 juillet 2021.

\section{cc) (1) 8}

Cahiers balkaniques est mis à disposition selon les termes de la Licence Creative Commons Attribution

- Pas d'Utilisation Commerciale 4.0 International. 


\title{
Kazantzakis, un intellectuel à l'épreuve de la réalité
}

\author{
Kazantzakis, an Intellectual confronted to Reality

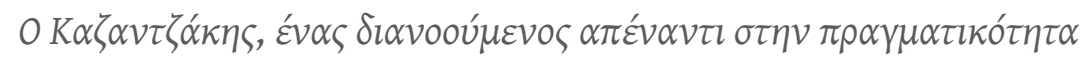

\author{
Gunnar De Boel
}

\section{Kazantzakis : un « bolcheviste » dans le réseau clientéliste libéral}

1 Kazantzakis est un intellectuel pur-sang. Tout en pratiquant les belles lettres dès l'âge de 23 ans, il rend compte de ses multiples voyages en écrivant des correspondances pour des journaux athéniens et gagne son pain en écrivant des livres scolaires et des articles d'encyclopédie pour les éditeurs athéniens Dimitrakos et Elefthéroudakis.

2 Il fait clairement partie du réseau clientéliste du parti libéral, venizéliste (BIEN, 2007, 53). De la même façon qu'il pourra toujours compter sur le soutien de Georgios Papandréou (JANIAUD-LUST, 1970, 477 ; BIEN, 2007, 51, 55, 56), figure de proue de ce parti, sur celui d'auteurs libéraux comme Théotokas (BIEN, 2007, 217 ; du moins jusqu'en 1946, cf. TZIOvAs, 2006, 80) et, bien sûr, Prévélakis qui lui reste aussi acquis. Au contraire, quand les monarchistes sont au pouvoir, Kazantzakis n'a rien à espérer des pouvoirs publics. La décennie des années 1920, pendant laquelle il se déclare pourtant " bolcheviste de l'extrême " $^{1}$, dans une lettre de 1923 (1958, 152), à sa première épouse, Galateia, ne change rien à son appartenance au réseau venizéliste, même si cela le place dans un grand écart avec lui-même. Ainsi, dans une autre lettre à Galateia, de 1922 (1958, 90), il appelle le coup d'état du colonel Plastiras, après la débâcle de l'armée grecque en Asie Mineure, "notre révolution », parce qu'elle est le fait d'un venizéliste, même s'il se demande immédiatement après si cette révolution émane du peuple ou de certaines personnalités qui se servent des masses; il conclut: "C'est toujours une lumière, un pâle reflet du grand incendie futur, du nôtre $»^{2}$. 

surtout en grand intellectuel, qui essayait aussi d'écrire :

Voilà ma force : la pensée et l'écriture. La première, je la réussis bien, je n'ai pas honte de le dire. Je sens mon cerveau bien solide, de très bonne qualité, très lumineux. La deuxième, l'écriture, je la réussis moyennement. Mon art n'est pas net, pur, grand.

$(1958,127)^{3}$

\section{Penseur ou écrivain?}

5 Or, il est permis de douter du jugement qu'il porte sur sa carrure de penseur. Il est certain que Kazantzakis avait beaucoup lu, mais il est aussi certain qu'il était un grand compilateur. On sait que la théorie métaphysico-philosophique qu'il a consignée dans son Ascèse $e^{4}$ était surtout un mélange de l'élan vital de Bergson et du Surhomme de Nietzsche. Mais dans sa thèse sur Nietzsche, qu'il a écrite pendant son séjour parisien de 1907 à 1909, son accès à ce philosophe était encore indirect : en effet, il recopiait de façon éhontée des pages entières des commentateurs français de Nietzsche, en particulier Henri Lichtenberger, Émile Faguet et Alfred Fouillée (DE BOEL, 2006). Cela l'amenait, par exemple, à adopter l'idée de Lichtenberger, selon lequel Nietzsche enseignait que « l'homme est une parcelle de la volonté éternelle et infinie, et en cette qualité, il est, lui aussi, éternel et indestructible» (1899, Ix). Trois ans plus tard, Lichtenberger ira jusqu'à dire que Nietzsche enseigne que «l'homme est une parcelle de la substance infinie, de la divinité » $(1902,783)$.

Kazantzakis base sur cet enseignement, attribué de façon erronée à Nietzsche, son idée

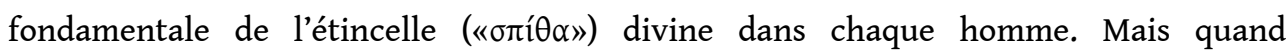
Nietzsche parle de "vie éternelle ", c'est uniquement pour se moquer du christianisme, qui utilise la croyance en une vie éternelle comme os à ronger pour détourner l'attention de cette haine pour la vie et de cette qualité misérable de la vie qu'il prêche (DE BOEL, 2009, 116-120). Nous verrons que sur ce point, loin de s'aligner sur Nietzsche, Kazantzakis se trouve plutôt sur la ligne du christianisme le plus ascétique, le plus hostile à la vie terrestre. Cette ligne-là, le véritable Nietzsche la tenait en horreur. Le compilateur se laisse donc piéger par les sources secondaires qu'il utilise trop facilement.

\section{Idéologie ou métaphysique?}

7 Dans une lettre de 1936 à son ami Prévélakis, Kazantzakis divise sa vie jusque-là en trois périodes, du point de vue de l'idéologie à laquelle il adhérait alors :

- Jusqu'en 1923, il était nationaliste, et sentait à côté de lui l'ombre de Dragoumis.

- De 1923 à 1933, il suivait la gauche (sans n'avoir jamais été communiste, ajoute-t-il), et sentait à côté de lui l'ombre de Panaït Istrati.

- Maintenant, il ne suit plus personne ni aucune couleur: ce dernier stade est celui de la liberté (PRÉVÉLAKIS, 1965, 464-465).

8 Mais il faut se méfier quand un auteur fait lui-même l'analyse de son œuvre et de sa carrière d'écrivain ou de penseur. D'une part, ces coupures sont loin d'être absolues. Il 
est évident que pendant la décennie de 1930 l'ombre de Dragoumis est toujours bien là (BIEN, 2007, 131; 158). D'autre part, aucune période n'est exclusivement dominée par une seule idéologie. Ainsi, il avait, dès le début de sa période de gauche, une appréhension fondamentale devant le côté matérialiste de la doctrine socialiste. Enfin, il est évident que pendant la troisième période, comme déjà pendant la décennie précédente, le fascisme, dans ses manifestations italienne et espagnole, attire notre auteur. Déjà en 1924, il dit dans une lettre à son épouse que « Mussolini est peut-être beaucoup plus grand que ce que nous avions coutume de croire jusqu'ici» $(1958,235)^{5}$. C'est précisément le côté totalitaire du fascisme qui le fascine: la mainmise d'un mouvement non seulement sur le cadre politique, mais sur tout l'environnement social, économique et culturel de la société, exactement comme ce qui se passe avec le bolchevisme (ibidem). En 1936, il décrit, dans le texte la Peur et la Faim ${ }^{6}$ auquel nous aurons à revenir, la gauche et le fascisme comme des

collaborateurs secrets, qui se battent pour le même but.

- Quel but?

- Que l'âme de l'homme se secoue un peu, qu'elle reste éveillée autant qu'elle peut, qu'elle tremble un peu de peur, ou qu'elle se rue tout autour, insatiable, poussée par la faim.

(VRETTAKOS, 1960, 579) ${ }^{7}$

9 Aussi, tout porte à croire que ces différents changements de cap sont surtout superficiels, et que le seul cap qu'il gardera pendant toute sa vie et qui le fera toujours prendre le côté des idéologies les plus extrémistes, c'est son aversion des valeurs bourgeoises telles que la stabilité, le confort, le bonheur (BIEN 2007, 35).

10 Ainsi, dès son manifeste Ascèse il reprend à son compte les exhortations de Dieu, qui ordonne de brûler maison et idées, et d'abandonner femme et enfants pour le suivre (1965, 76-77). C'est clairement cet aspect-là du Jésus néotestamentaire (cf. p. ex. l'évangile de Luc, 18, 29-30) qui inspire Kazantzakis.

11 Dans son évangile à lui, Kazantzakis ne cesse de prêcher la haine. Il déclare la guerre à ceux qui sont contents, rassasiés, stériles. La haine vaut mieux que la philanthropie essoufflée, parce qu'il faut rechercher l'impossible, comme le font les amoureux (1965, 77-78). Par un tour de passe-passe bien typique, l'amour devient donc synonyme de la haine. L'humanité est appelée à suivre l'exemple du Dieu de Kazantzakis, qui n'est pas précisément un Dieu de bonté. Il est dur, et ne soucie pas des êtres humains ou des animaux : il crée tout, l'aime un instant et puis l'anéantit $(1965,66-67)^{8}$. Il y a donc un travail de destruction à faire : il faut faire table rase de ce qui existe, car essayer de seulement l'améliorer ne ferait qu'aggraver le mal. C'est pourquoi il faut d'abord augmenter l'injustice, pour pouvoir tout anéantir ensuite et recommencer sur des bases saines $(1965,78)$.

\section{La grille de lecture appliquée au monde réel}

Ce qu'il veut dire par là, à un niveau non plus métaphysique, mais pratique, est expliqué clairement dans une lettre à Galateia datant de mai 1923, contemporaine à l'écriture d'Ascèse :

Toute tentative d'amélioration diffère la victoire définitive. Voilà encore une invention, une ruse de bourgeois déguisés. Aujourd'hui, nous n'avons qu'un seul devoir : le Renversement. (...) Le « Renversement » doit dépasser ce premier stade, celui de la discussion, et entrer dans le deuxième - le stade de la foi, sur laquelle 
plus aucune discussion n'est possible; tout ce qu'elle fait, c'est d'allumer les cœurs, d'engendrer des actions, de détruire des civilisations et d'en créer de nouvelles. Jamais les discussions n'ont renouvelé la face de la terre. (...) Ce qui a toujours renouvelé la Terre c'est la Passion, l'enthousiasme, la Foi sans arguments intellectuels.

$(1958,202-203)^{9}$

Ce rejet de la discussion, des arguments intellectuels, rationnels, et cet éloge de la Foi sont des caractéristiques qui peuvent étonner de la part d'un intellectuel. En fait, qu'il soit inspiré - comme cela lui est arrivé successivement-, par le nationalisme, le communisme ou le fascisme, la seule chose qui importe vraiment pour Kazantzakis, c'est de détruire de fond en comble la civilisation bourgeoise existante: celle de l'Europe occidentale, surtout de la France et de la Grande-Bretagne. Voilà à ses yeux les responsables de tout ce qui va mal. Ainsi, quand en 1936 des intellectuels appellent à signer une pétition contre l'invasion de l'Éthiopie par l'Italie mussolinienne, il publie, le 20 juillet 1936, dans le journal Kathimerini le fameux texte la Peur et la Faim, où il dit :

Parmi les grandes nations, l'Allemagne, le Japon, l'Italie suffoquent aujourd'hui dans leurs frontières, elles ne peuvent pas s'étendre, elles ont faim; la France et l'Angleterre sont rassasiées, elles ont partagé le monde entre elles et regardent avec peur les peuples affamés, sans terre. (...) Il y a quelques mois, nos «intellectuels » ont colporté une pétition furieuse et indolore contre l'Italie qui s'est jetée sur l'Abyssinie pour la manger. Quelqu'un m'a demandé si je la signerais. (...) Je la signerais, si la protestation était contre l'Angleterre et la France, qui possèdent le monde entier et refusent de donner de la terre aux peuples pauvres pour qu'ils puissent vivre. Ce sont les capitalistes inhumains, rassasiés, parmi les nations, et ils ne laissent pas les autres peuples, les prolétariens, lever la tête.

(VRETTAKOS, 1960, 577-578) ${ }^{10}$

14 La destruction totale de cette civilisation de nantis est nécessaire. On en a observé les bienfaits, dit Kazantzakis, dans une lettre envoyée à sa femme le lendemain de l'évacuation de Smyrne par l'armée grecque, en septembre 1922, en Allemagne et en Russie, où la destruction suite à la défaite dans le premier cas, et à la révolution bolchevique dans le second, a conduit à une renaissance de ces pays, contrairement à la France. De la même façon, il espère que le désastre qui secoue la Grèce à ce moment précis sera le début de sa renaissance :

C'est comme ça que je le prends, et je l'accepte avec gratitude. Une victoire pour le régime actuel serait néfaste pour la Grèce. Elle mettrait bien en selle les infâmes actuels et elle anesthésierait le peuple, qui ne demande que ça. Ce grand malheur, au contraire, ou bien le fortifiera, ou bien l'anéantira. Les deux issues sont préférables à son existence misérable actuelle.

$(1958,77-78)^{11}$

15 Ces paroles glaciales semblent annoncer la conception hitlérienne, pour qui le peuple allemand méritait sa destruction, vu qu'il s'était montré incapable de vaincre.

Remarquons tout de même en passant que, dans la vraie vie, la haine n'inspire qu'horreur à Kazantzakis, pour qui la campagne d'Asie Mineure révèle les limites du chauvinisme. Ainsi il écrit à Galateia, depuis Belgrade, en mai 1922 :

Quelle horreur que toutes ces frontières, remplies de haine et de sang. (...) Je pense aux Turcs, aux Bulgares, à tous ces gens qu'on nous a appris à haïr. Quelle douceur dans mon cœur maintenant !

$(1958,16)^{12}$

Mais il ne faut pas oublier que Kazantzakis ne se soucie pas des individus : le lendemain de l'évacuation de Smyrne, il écrit à sa femme : «L'Homme nous fend le cœur, c'est 
pour lui que nous nous battons, pas pour les hommes minables» $(1958,77)^{13}$. L'internationalisme affiché ne change en rien la priorité toujours donnée à la destruction. En ce qui concerne le programme de reconstruction, Kazantzakis ne propose rien de bien concret : il dit - toujours dans une lettre à Galateia ! - que l'école doit préparer les élèves à un seul but :

[...] qu'ils détruisent le monde ancien (celui de leurs parents) et qu'ils en construisent un nouveau. Le but de l'École doit maintenant être clair, impitoyable, strict. Plus tard, plus tard, quand nous aurons vaincu, avec la force de notre Dieu, alors nous verrons. Alors viendra l'art, la musique, l'idylle. Et si nous vivions à ce moment-là, nous serions les premiers à poser à nouveau des mines, pour une nouvelle destruction et une nouvelle création.

$(1958,174)^{14}$

En d'autres termes, ce moment de la reconstruction ne viendra jamais. La destruction doit être permanente, et elle doit concerner aussi - surtout - la culture, tout comme la révolution maoïste - que d'ailleurs, bien sûr, il admirera plus tard (1962, 323 ; 326).

\section{Les « savants barbares »: Tolède, Londres}

Ce travail de destruction est confié, dans la vision de Kazantzakis, aux peuples barbares. À l'origine, il pensait que les Russes communistes s'en acquitteraient, mais il se rend compte que ceux-ci s'embourgeoisent très vite. Toutefois, au cours des années 1930 il voit, émerveillé, apparaître des savants barbares dans la guerre d'Espagne, qui utilisent la force industrielle pour détruire des villes. Il regarde étonné ses collègues journalistes, qui suivent le spectacle de cette destruction sans la moindre détresse. Cette absence de détresse, il l'a d'ailleurs déjà diagnostiquée en lui-même, au moment où il observe des bombardiers qui lâchent leurs bombes sur Tolède :

$\mathrm{Au}$ lieu d'anges, des avions maintenant, mais le but mystique était le même : que Tolède se secoue, se libère de la certitude, de la sagesse et de la médiocrité, qu'il devienne cendres, haute vision, témoin tout pâle, tout blessé d'une idée. Que l'idée mange le corps, que celui-ci n'existe plus pour rendre trouble la flamme. La substance de Tolède est restée, tout le superflu est devenu cendres.

J'ai honte de l'écrire, mais je n'ai senti aucune détresse. Au contraire. Une joie sauvage m'a saisi. Ce Tolède-ci est plus utile à l'homme que l'autre Tolède qui m'avait tellement déçu quand je l'ai vu la première fois. Je m'attendais à voir un rocher sans eau, sans verdure, avec des hommes secs et taciturnes. Et j'avais trouvé une ville riante, provinciale, avec des commerçants, des photographes, des popes. Maintenant, ils sont encore tous là - leur espèce ne s'élimine pas si facilement que ça-, mais dans leurs yeux se reflètent les ruines et les terribles scènes qu'ils ont vues et les angoisses qui sont passées; et cela les rend un peu moins commerçants, photographes et popes.

$(1964 a, 164)^{15}$

20 "Que Tolède se secoue...». Ce texte date du séjour de Kazantzakis comme correspondant pour le Kathimerini en Espagne, d'octobre à novembre 1936. On reconnaît tout de suite la définition qu'il avait donnée, en juillet de la même année, dans la Peur et la Faim, du but - toujours cette terrible perspective téléologique ! commun pour lequel, d'après lui, aussi bien la gauche que le fascisme se battent : «que l'âme de l'homme se secoue un peu" (voir plus haut). On se rend compte que sa description du bombardement de Tolède est dans la droite ligne de ce qu'il écrivait dans son Ascèse à propos des gens " contents, rassasiés, stériles ». Tolède est coupable d'un bonheur mesquin: les gens rient, en plus d'être stupidement commerçants, 
photographes, curés. Le bombardement a donc "sauvé», délivré la ville de ses certitudes, de sa sagesse, de sa médiocrité. L'emploi de mots clés d'Ascèse, comme

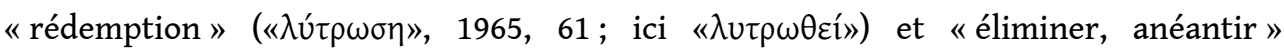

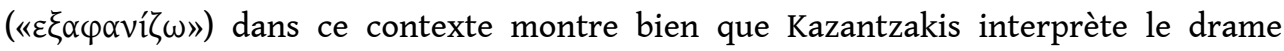
espagnol selon les catégories propres au système métaphysico-religieux développé dans son Ascèse. L'important est de comprendre que ce livre n'est pas seulement un traité de métaphysique, déconnecté de la réalité de tous les jours. Il forme au contraire la grille à travers laquelle les événements sanglants d'une guerre bien réelle sont interprétés !

Et ce n'est pas fini. Kazantzakis se trouve à Londres, en 1940, quand les premiers bombardiers allemands survolent le pays; il se réjouit de voir à nouveau des savants barbares qui vont ravager les villes :

À ce moment-là, on entendait hurler les toutes premières sirènes d'alarme à Londres. (...) Les sirènes signalent l'approche des avions ennemis, les clochettes les gaz toxiques et les maladies que le savant barbare de nos jours a appris aux hommes à répandre pour ravager les villes. (...)

Une curiosité sauvage, inhumaine faisait en sorte que je reste immobile. Voilà, me dis-je, le premier cri, le premier râle de la civilisation industrielle mourante. Le mot d'ordre de la destruction vient d'être donné. (...)

Jusqu'à maintenant, songeais-je, je n'ai jamais vécu un instant aussi crucial à l'échelle mondiale. Il faut que j'en profite jusqu'au bout !

(1964b, 220-221) $)^{16}$

Ce texte sera publié début 1941, au moment où la nation grecque repousse l'envahisseur italien. Kazantzakis travaille encore à son roman Au palais de Cnossos ${ }^{17}$, qu'il destine à la Jeunesse ${ }^{18}$, le magazine du mouvement de la jeunesse EON du régime dictatorial de Metaxas. Ce roman, dans le droit fil de l'Odyssée de l'auteur, représente les puissances coloniales, la Grande-Bretagne et la France, sous les couleurs de la civilisation minoenne décadente (BEATON, 2006, 184). Ces démocraties libérales sont les

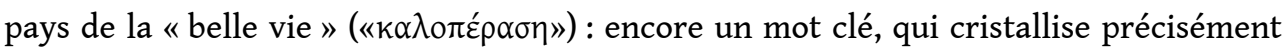
l'horreur de Kazantzakis devant le confort de la vie bourgeoise. Ce mot est associé à l'Angleterre, de façon apparemment anodine, dans la relation de son voyage en Angleterre : le goût pour la belle vie constitue la cohésion profonde de la civilisation anglaise $(1964 b, 73)$. Mais le même mot est utilisé dans Au palais de Knossos, où il caractérise justement la décadence de cette civilisation épuisée, qui a oublié comment se battre $(2007,326)$. La clé de l'interprétation est donnée une fois de plus par Ascèse, où ce mot apparaissait dans un contexte qui rappelle le Discours sur la Montagne de Jésus (Mt. 5). Il y est dit que Dieu n'est pas un bon père de famille équitable :

L'Injustice, la Dureté, le Désir, la Faim sont les quatre juments qui dirigent son char sur notre terre accidentée.

Dieu n'est jamais créé à partir de bonheur, de belle vie et de gloire, mais à partir de honte, de faim et de larmes.

$(1965,75)^{19}$

Dans Au palais de Cnossos, cette civilisation de la «belle vie » sera détruite, à la fin du livre, par les barbares Doriens, qui ressemblent comme deux gouttes d'eau aux Russes soviétiques (KAKRIDIS, 2005, 284-285; DE BOEL, 2014, 271-272). Heureusement pour Kazantzakis, la publication du magazine est arrêtée après l'invasion allemande en Grèce, et le roman, que l'auteur avait soigneusement oublié dans son tiroir, ne sera publié qu'en 1981. 


\section{L'écrivain se libère du penseur}

24 allemands a rétabli les relations si difficiles de Kazantzakis avec son propre peuple, qu'il commence à estimer et à aimer, et qu'il voit souffrir si désespérément pendant le premier hiver de l'occupation (BIEN, 2007, 162). Là, il n'est plus question de se réjouir de la guerre. Déjà, quand il voit les bombardiers allemands survoler son île d'Égine, le 15 mai 1941, ce n'est plus de la fascination qu'il ressent, mais de l'inquiétude, et il exhorte Eleni, sa future deuxième épouse de supporter avec dignité ces moments terrifiants $(1968,396)$. Malgré la prise de conscience, annoncée dès 1922, que la souffrance de membres d'autres peuples le touche désormais autant que la souffrance d'un Grec, malgré ces professions d'internationalisme, c'est bien la souffrance de son propre peuple qui fait le déclic en 1941. Désormais, la guerre est condamnée, dans le roman Zorba (BIEN, 2007, 164), qu'il commence à écrire en août 1941 et qu'il termine en mai 1943. Ce roman est un règlement de comptes tardif avec Dragoumis (BIEN, 2007, 161), le chef de l'action grecque en Macédoine ottomane. Zorba a participé à cette luttelà, et il n'en est pas fier :

Autrefois, je disais : celui-ci est Turc, ou Bulgare, celui-là est Grec. J'ai fait des choses pour la patrie, patron, qui te feraient dresser les cheveux sur la tête: j'ai égorgé, j'ai volé, j'ai brûlé des villages, j'ai violé des femmes, j'ai anéanti des maisons... Pourquoi ? Parce qu'ils étaient Bulgares, Turcs (...) Je suis devenu plus malin, je regarde maintenant les gens et je dis : celui-ci est quelqu'un de bien, celuilà un méchant. Ça n'a pas d'importance s'il est Bulgare ou Grec. C'est pareil pour moi. Bon ou méchant, voilà la seule question que je me pose maintenant. Et comme je vieillis, (...), je crois que je commence à même plus me poser cette question-là ! (...) J'ai pitié de tous (...).

$(1959,268)^{20}$

Kazantzakis semble découvrir dans ce roman les horreurs de la guerre, en même temps qu'il célèbre l'être humain individuel avec ses petits bonheurs mesquins de la vie de tous les jours, ceux-là précisément qui désignaient la ville de Tolède comme ville «à anéantir ». L'intellectuel, toujours en vadrouille dans le monde entier, toujours à l'affût des bouleversements qui « secouent » les sociétés, tout en restant de marbre devant les souffrances des individus, a été finalement touché en plein cœur par le spectacle de l'héroïsme d'abord, de la grande misère ensuite de ses compatriotes, qu'il ne pouvait plus traiter juste comme des pions dans la marche du monde. Finalement, le penseur plutôt médiocre, mais fanatique peut utiliser ses dons littéraires indéniables à bon escient, et devenir un grand écrivain.

\section{BIBLIOGRAPHIE}

BEATON Roderick, 2006, "Minoans in Modern Greek literature”, in Giannis HAMILAKIS \& Nicoletta MOMIGLIANo (éds), “Archaeology and European Modernity: Producing and Consuming

Cahiers balkaniques, Hors-série | 2015 
the 'Minoans': Creta Antica", Rivista annuale di studi archeologici, storici ed epigrafici, Centro di Archaeologia Cretese, Università di Catania, vol. 7, pp. 183-95.

BIEN Peter, 2007, Kazantzakis, Politics of the Spirit, vol. 2. Princeton \& Oxford: Princeton University Press.

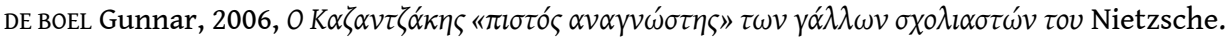
Athènes : Psychogios (éd.), p. 215-228.

DE BOEL Gunnar, 2009, “The French sources between the young Kazantzakis and Nietzsche”, The Historical Review/La Revue Historique, vol. v, pp. 107-120.

DE BOEL Gunnar, (2014), "Rethinking the Greek Legacy. Dorians in Modern Greek Fiction”, in D. TZIOVAS (éd.), Re-Imagining the Past. Antiquity and Modern Greek Culture, Oxford: Oxford University Press, pp. 266-281.

JANIAUD-LUST Colette, 1970, Nikos Kazantzaki, Sa vie, son œuvre (1883-1957), Paris : François Maspero.

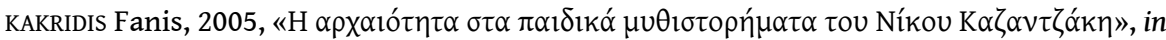

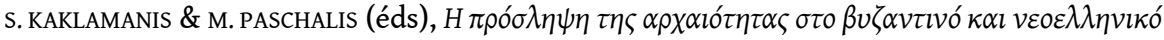
$\mu v \theta \imath \tau \tau o ́ \rho \eta \mu \alpha$, Athènes : Stigmi.

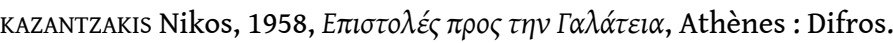

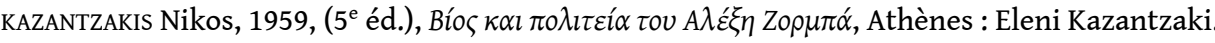

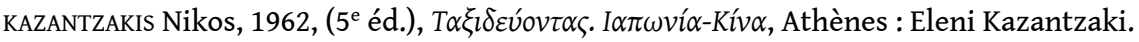

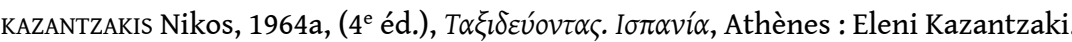

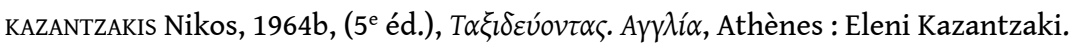

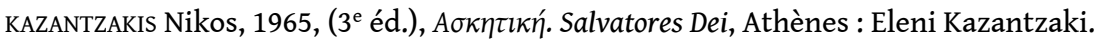

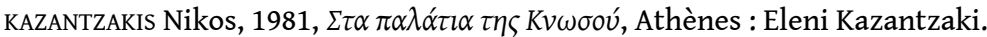

LICHTENBERGER Henri, 1899, Friedrich Nietzsche. Aphorismes et fragments choisis, Paris : Félix Alcan.

LICHTENBERGER Henri, 1902, « Le testament philosophique de Nietzsche », La Revue de Paris, vol. 9, $\mathrm{n}^{\circ} 2$, p. $779-808$.

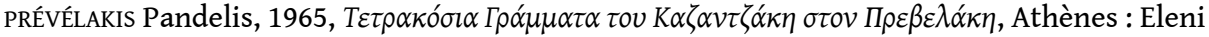
Kazantzaki.

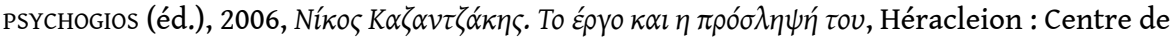
littérature grecque.

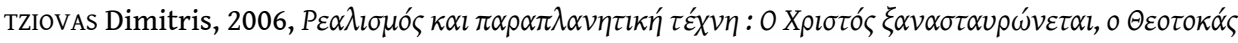

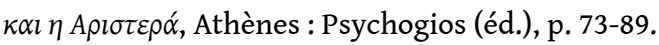

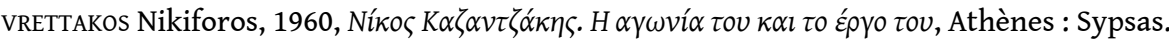

\section{NOTES}

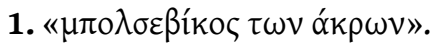

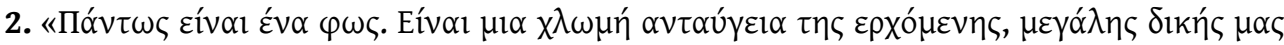
$\pi u \rho \kappa \alpha \gamma i \alpha ́ \alpha »$. 


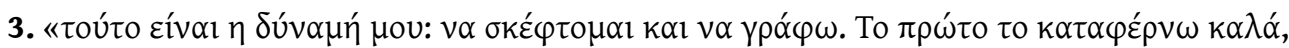

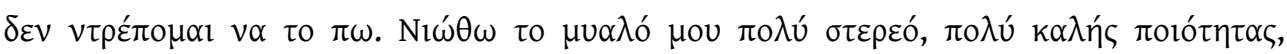

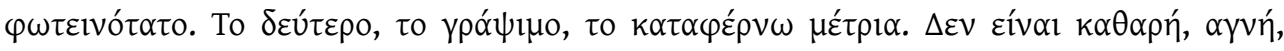

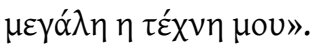

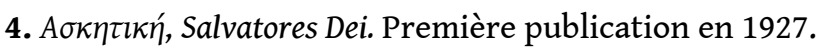

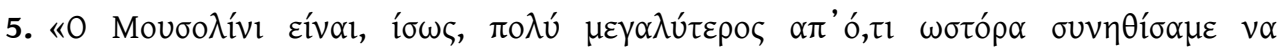

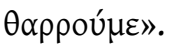

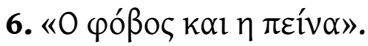

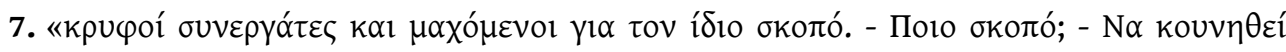

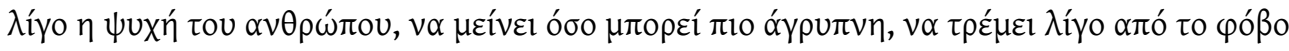

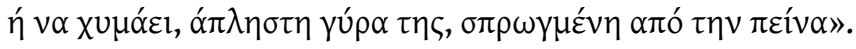

8. Le verbe utilisé est $\alpha \varphi \alpha v i ́ \zeta \varepsilon l$. Nous aurons l'occasion de le retrouver dans des contextes moins métaphysiques.

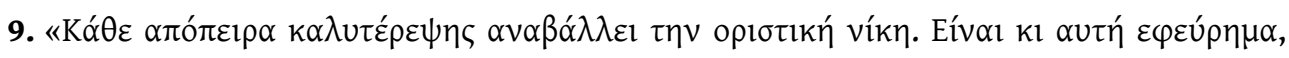

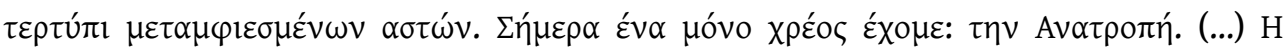

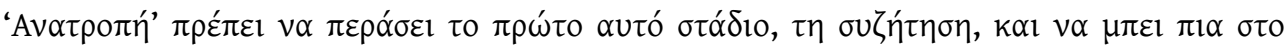

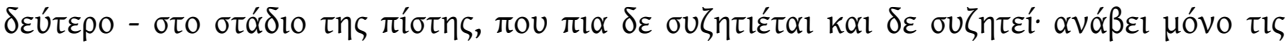

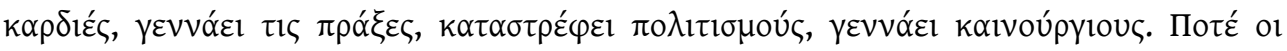

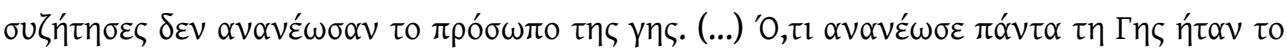

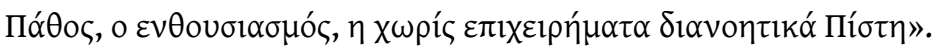

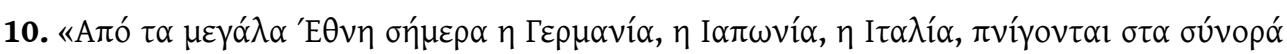

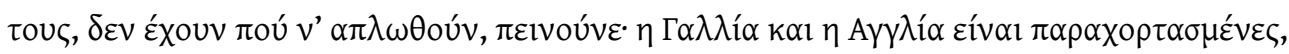

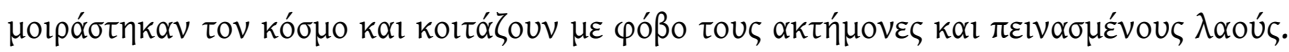

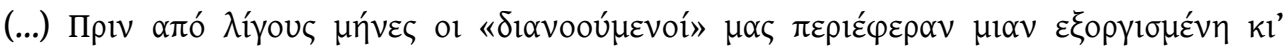

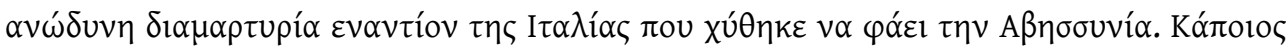

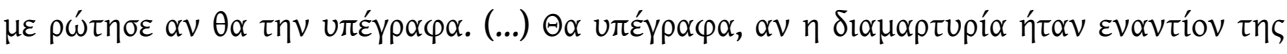

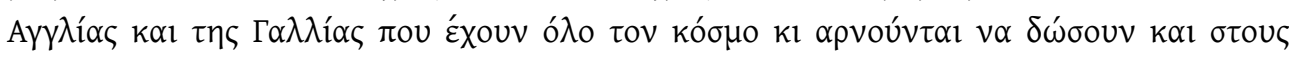

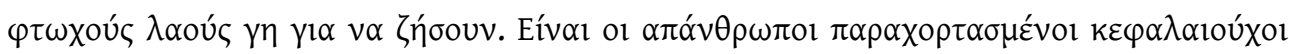

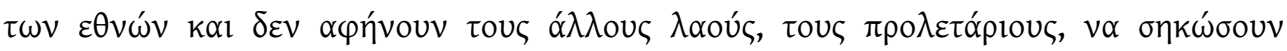

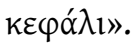

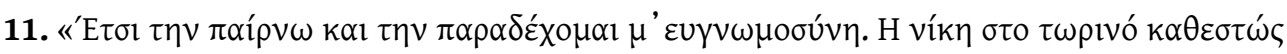

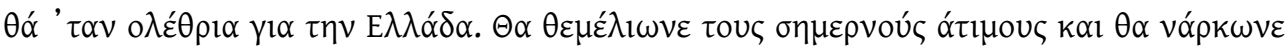

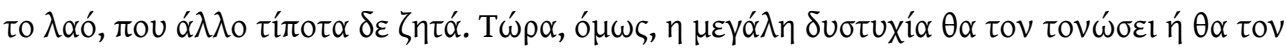

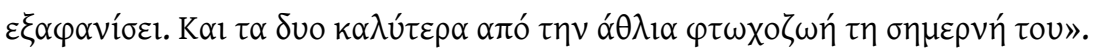

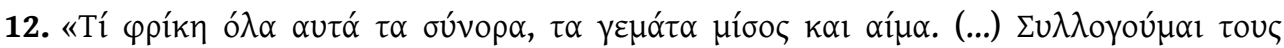

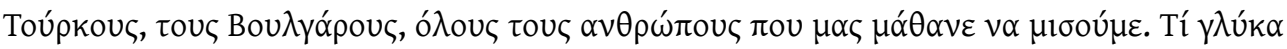

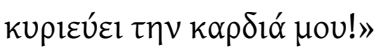

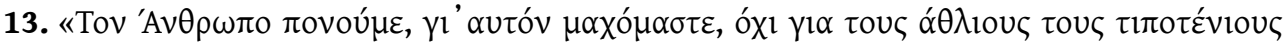
$\alpha v \theta \rho \omega ́ \pi$ rovৎ».

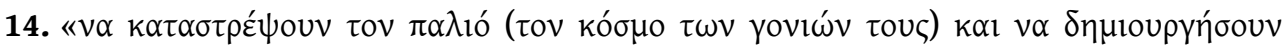
kalvoúpyio».

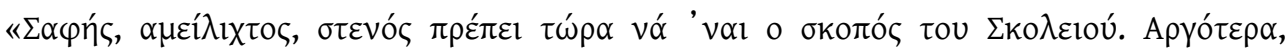

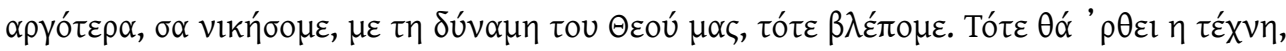




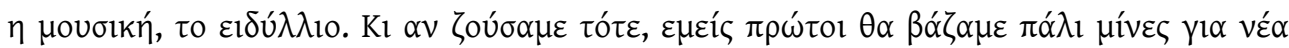

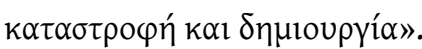

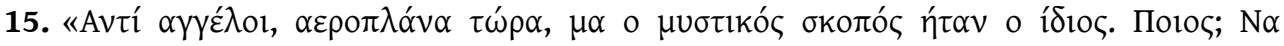

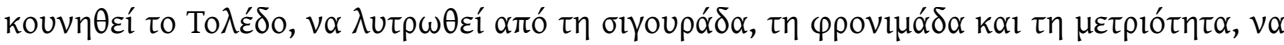

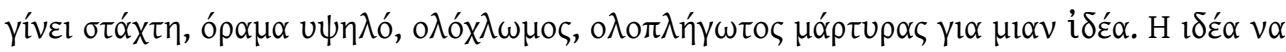

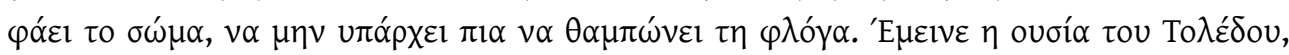

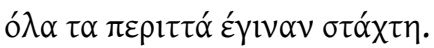

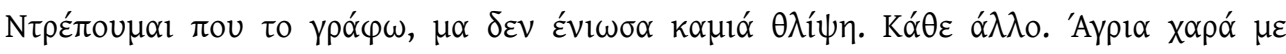

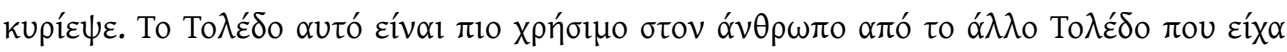

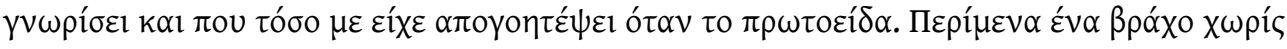

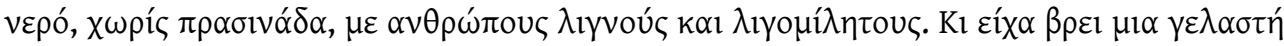

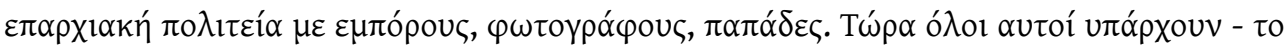

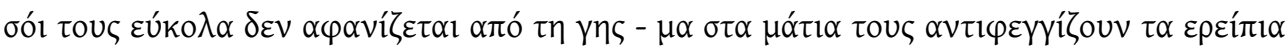

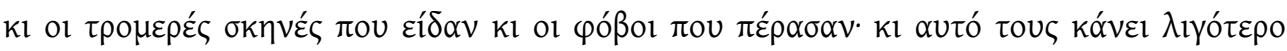

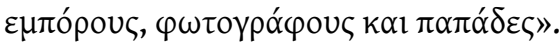

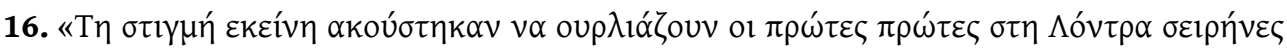

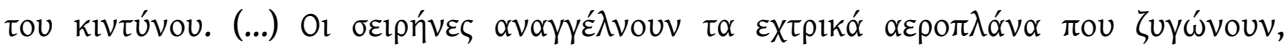

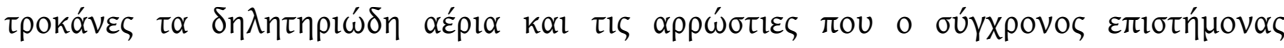

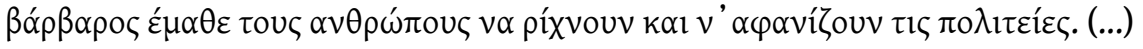

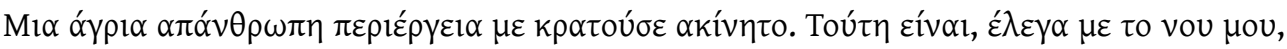

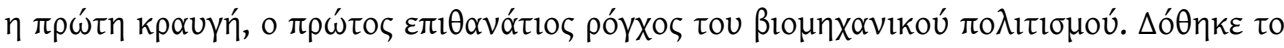

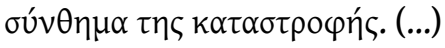

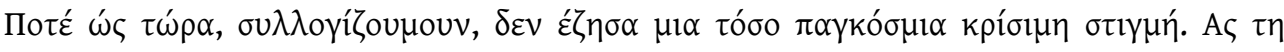

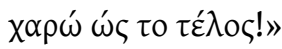

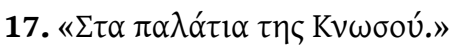

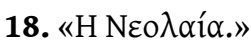

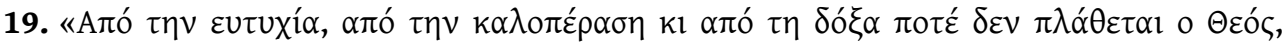

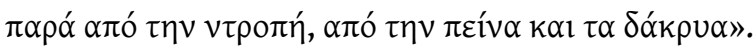

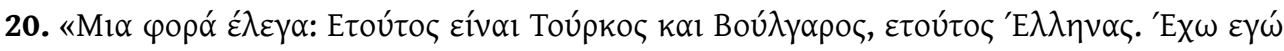

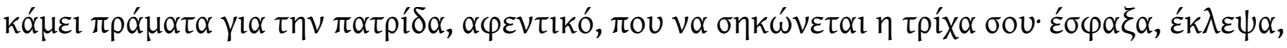

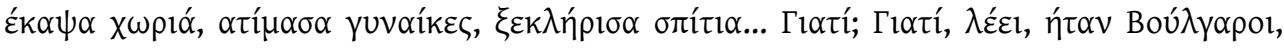

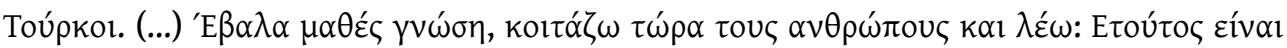

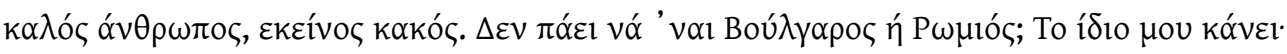

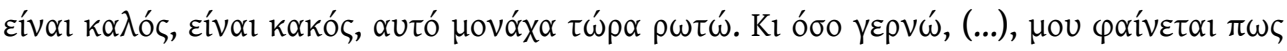

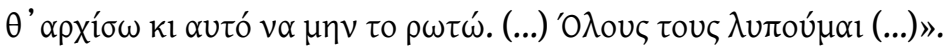

\section{RÉSUMÉS}

Pour Kazantzakis, Ascèse n'est pas qu'une métaphysique abstraite ; ses idées anti-humanistes sont utilisées aussi pour interpréter la réalité contemporaine. Ce n'est que confronté à la misère qui 
en résulte quand elles sont appliquées dans la réalité, qu'il les abandonne et devient un écrivain véritable.

For Kazantzakis, Askitiki is not just an abstract metaphysics; its anti-humanistic ideas are also used to interpret contemporary reality. Only when he is confronted with the misery that results from their application in reality, he abandons them and becomes a real writer.

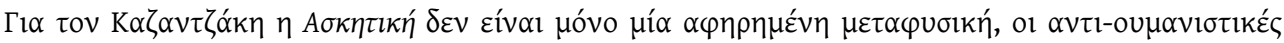

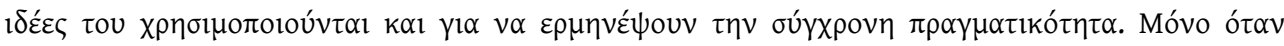

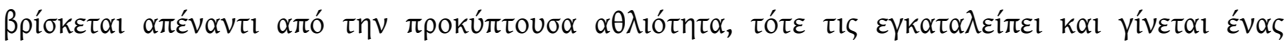

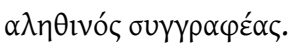

\section{INDEX}

Index géographique : Grèce

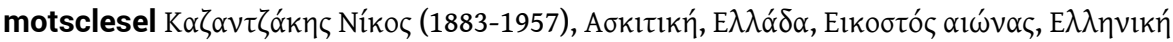

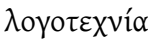

motsclesmk КАЗАНЬАКИС НИКОС (1883-1957), АСКЕТИЗАМ, ГРЦИЈА, ДВАЕСЕТТИОТ ВЕК, ГРЧКАТА ЛИТЕРАТУРА

Thèmes : Littérature grecque

Mots-clés : Kazantzakis Nikos (1883-1957), Kazantzakis Nikos (1883-1957)

motsclestr Kazancakis Nikos (1883-1957), Çileci (Askitiki), Yunanistan Yirminci yüzyılda Yunan Edebiyat 1

Keywords : Kazantzakis Nikos (1883-1957), Ascesis, Greece, Twentieth century, Greek literature Index chronologique : vingtième siècle

\section{AUTEUR}

GUNNAR DE BOEL

Université de Gand 